\title{
La diáspora china en México. Asociaciones chinas en el Distrito Federal, Mexicali y Tapachula
}

\section{The Chinese diaspora in Mexico: Chinese associations in the Distrito Federal, Mexicali and Tapachula}

\author{
SERGIO E. MARTÍNEZ RIVERA* \\ ENRIQUE DUSSEL PETERS**
}

\begin{abstract}
RESUMEN: Con base en entrevistas de más de una docena de asociaciones de inmigrantes chinos y sus descendientes en tres entidades de México (Distrito Federal, Mexicali y Tapachula), el documento concluye sobre un grupo de aspectos actuales de estas asociaciones. Destaca una comunidad china relativamente integrada a nivel local, pero limitada con la propia República Popular China, además de limitaciones en la promoción de proyectos económicos con los gobiernos locales y regionales. También llama la atención la existencia y convivencia de al menos cuatro agrupaciones de instituciones de inmigrantes chinos en México: las "históricas» resultantes de flujos migratorios desde Estados Unidos y directamente desde China, pero también un importante crecimiento de otras tres agrupaciones: instituciones de inmigrantes chinos de varias generaciones, instituciones de inmigrantes chinos con fines económico-comerciales y otro muy importante grupo de instituciones chinas vinculadas a la muy reciente «oleada» de inmigrantes chinos desde la primera parte del siglo XXI.
\end{abstract}

PALABRAS ClaVE: Inmigración, China, México, Distrito Federal, Mexicali, Tapachula, asociaciones chinas.

ABSTRACT: Based upon interviews with more than a dozen Chinese immigrant associations and their descendants within three states in Mexico (Distrito Federal, Mexicali and Tapachula), this study identified a number of current characteristics of these associations. It uncovers a Chinese community relatively integrated at the local level, but which has limited ties with the People's Republic of China, as well as limited promotion of economic projects among local and regional governments. The article also calls attention to the existence and cooperation among at least four institutionalized groups of Chinese immigrants in Mexico: «the historical» - the results of migratory flows from the United States and directly from China, but also a significant growth in three other groupings: institutions of Chinese immigrants across various generations; institutions of Chinese immigrants with economic-commercial goals; and another very important group of Chinese institutions linked to the very recent "wave» of Chinese immigrants from the early years of the 21 st Century.

KEY words: Immigration, China, Mexico, Distrito Federal, Mexicali, Tapachula, Chinese Associations.

*Profesor-investigador del Centro de Estudios China-México de la Facultad de Economía de la Universidad Nacional Autónoma de México (UNAM).

**Profesor del Posgrado en Economía de la Universidad Nacional Autónoma de México (UNAM) y Coordinador del Centro de Estudios China-México de la Facultad de Economía de la UNAM. 


\section{INTRODUCCIÓN ${ }^{1}$}

7 a República Popular China — en lo que sigue China - ha jugado un papel crecientemente relevante en México. No sólo se ha convertido en el segundo socio comercial de México desde 2003, sino que ha incrementado su presencia en términos económicos, políticos y culturales, entre muchos otros ámbitos. Destaca el reciente salto cualitativo en el relacionamiento político: si hasta 2012 la relación política había llegado a su peor nivel en las últimas décadas - y considerando el inicio de las relaciones diplomáticas desde el 13 de febrero de 1972 (Jiménez Macías, 2012)desde 2013 la relación ha mejorado significativamente. El presidente Enrique Peña Nieto se encontró con su contraparte Xi Jinping en Boao, el segundo realizó una visita de Estado a México a mediados de 2013, y ambos mandatarios volvieron a encontrarse a finales del mismo año en el marco del G20 en San Petersburgo, Rusia. Sin embargo, la relación binacional ha vuelto a dificultades y tensiones semejantes a las anteriores a 2013 desde mediados de 2015 (Monitor de la Manufactura Mexicana, 2015).

En el contexto anterior, las relaciones políticas y económicas - y especialmente el comercio-, han recibido una mayor atención en el análisis en el último lustro, ${ }^{2}$ aunque diversos aspectos de la inmigración china han estado relativamente ausentes en los análisis de la relación México-China en las últimas décadas — con contadas excepciones, como se verá posteriormente-.

${ }^{1}$ Documento elaborado para el proyecto «La diáspora china en México. Un análisis sobre la forma y las dimensiones del transnacionalismo» con la participación conjunta del Centro de Estudios Latinoamericanos de la Universidad de Miami y el Centro de Estudios China-México de la Facultad de Economía de la Universidad Nacional Autónoma de México. Este estudio es parte del proyecto comparativo "Organizaciones de inmigrantes chinos en América Latina. Sus características y vínculos transnacionales», coordinado por Ariel Armony y Alejandro Portes. Los autores son los responsables únicos de los contenidos del documento. Los autores están agradecidos por los diversos comentarios de los miembros del proyecto, particularmente con Ariel Armony y Alejandro Portes.

${ }^{2}$ El análisis y la reflexión por parte del Centro de Estudios China-México - a través de conferencias, estadísticas, publicaciones y otros medios - refleja en buena medida el énfasis y los intereses de investigación actuales en México, véase http://www.economia.unam.mx/ cechimex/index.php/es/

$112 \frac{\text { PRIMER SEMESTRE } 2016}{\text { MIGRACIÓN Y DESARROLLO NÚM. } 26}$ 
El presente documento tiene como objetivo examinar a las asociaciones de inmigrantes chinos en México. ${ }^{3}$ En la actualidad existen contadas investigaciones que aborden las características y evolución de estas asociaciones, incluyendo aspectos como miembros, fundadores, principales actividades y participación dentro de la vida social, política y económica en las provincias mexicanas, entre muchas otras. El análisis también contribuye a la comprensión de aspectos nodales de la comunidad china en México en diversos momentos históricos.

\section{Marco conceptual y metodológico}

El documento se integra en los diversos trabajos y conceptos desarrollados recientemente por Alejandro Portes, específicamente la conceptualización de las asociaciones de inmigrantes como una «institución transnacional» (Portes, Escobar y Walton, 2013), y múltiples estudios en torno de las implicaciones que estas instituciones transnacionales efectivamente tienen en los procesos de desarrollo de los respectivos países. Las «formas de transnacionalismo» (Portes, Escobar y Walton, 2013: 267) dependen, entre otros factores, de la intervención estatal, la extensión y grados de formalización de las actividades y membresía en los países receptores, así como de diversas características de los contingentes de inmigrantes y las respectivas sociedades de origen: procedencia de la inmigración (rural/urbano), presencia de instituciones públicas (en algunos casos proactivas), extracción social y nivel educativo de los miembros, y costos de la propia inmigración. Como resultado, "los contextos de salida y recepción determinan el origen, fortaleza, y carácter de las organizaciones transnacionales» (Portes, Escobar y Walton, 2013: 276), existiendo significativas diferencias cualitativas y cuantitativas según las respectivas nacionalidades y sitios de destino. Zhou y Lee (2015), en su análisis de la inmigración china en Estados Unidos, destacan

${ }^{3}$ Futuros esfuerzos bien pudieran ampliar el universo de instituciones incluyendo por ejemplo las de Taiwán, que también juegan un papel relevante en el comercio entre México y China. 
aspectos como la independencia de las instituciones de inmigrantes chinos de las instituciones públicas chinas, así como funciones tanto de integración al país receptor como de vínculos con China. La función que estas instituciones de migrantes pueden jugar en el desarrollo de las regiones emisoras (Portes y Zhou, 2012) es un elemento que apenas se ha empezado a examinar con mayor detalle.

La recolección de datos para el análisis que sigue fue facilitada por la experiencia, lazos y el contacto que el Centro de Estudios China-México de la Facultad de Economía de la Universidad Nacional Autónoma de México (UNAM) mantiene desde hace más de una década con la comunidad china, empresas, líderes de opinión y diversas instituciones. Diversas publicaciones - particularmente los directorios de empresas e instituciones y la de diversos investigadores (Cechimex 2013; Martínez Rivera, 2012)— resultaron igualmente útiles.

Metodológicamente, este documento parte de los esfuerzos realizados por el Centro de Migración y Desarrollo de la Universidad de Princeton y el Proyecto «Organizaciones comparativas de inmigrantes». El formato desarrollado para ese proyecto fue adaptado a las condiciones específicas de las instituciones de inmigrantes chinos en México. Se realizaron múltiples entrevistas con instituciones chinas que no son, estrictamente hablando, de inmigrantes, así como con miembros de la comunidad china - empresarios, funcionarios, académicos y otros-, pese a carecer de membresía institucional, resultan relevantes para comprender las características generales de la comunidad china y sus asociaciones de inmigrantes en las últimas décadas. Partiendo de un universo de alrededor de 60 instituciones de inmigrantes chinos en México, el análisis se basa en un trabajo de campo (entrevistas y encuestas) que cubrió 12 asociaciones de inmigrantes chinos en las ciudades de Mexicali, Baja California, Distrito Federal y Tapachula, Chiapas.

Se seleccionaron estas ciudades y zonas aledañas considerando la importante presencia — histórica y actual — de la comunidad china en México, así como por la detección de instituciones de inmigrantes chinos. Para cada institución, la encuesta buscó identificar las principales características organizacionales (antigüedad, número de miembros, principales actividades, 
etcétera), así como los vínculos con su entorno nacional y con China en rubros como el cultural, político, económico, entre otros. A ello se agregó una revisión bibliográfica y entrevistas con académicos, expertos y líderes de la comunidad china en las respectivas regiones; la mayoría de estas entrevistas fue procesada separadamente de las encuestas debido a que los entrevistados no son miembros de instituciones de inmigrantes.

Se consideró como una «institución de inmigrantes chinos» a toda agrupación organizada formal o informalmente con el objetivo de realizar actividades de carácter cultural, educativo, político o económico, entre otros, y que incluyera entre sus objetivos formales o informales funciones para con los inmigrantes chinos. ${ }^{4}$ Varias de las instituciones encuestadas no reciben un reconocimiento jurídico o político (ya sea del gobierno central chino y/o por parte de las autoridades mexicanas) y operan a nivel local, regional, nacional y/o internacional. De igual forma, nos pareció importante integrar a la encuesta instituciones con miembros chinos residentes, nacionalizados como mexicanos o descendientes de migrantes chinos de diversas generaciones. Es decir, no se integró sólo a la comunidad e instituciones legalmente considerados como chinos - China sólo permite una nacionalidad-, sino que también a los esfuerzos y respectivas instituciones de comunidades chinas ya sea por definición propia y/o por sus características culturales y/o étnicas. El acceso y contacto con estas instituciones fue complejo y en varios casos las contrapartes accedieron a entrevistas, pero no a ser encuestadas con la información que se les requirió.

\section{Asociaciones chinas en México: ANTECEDENTES Y ACTUALIDAD}

De acuerdo con el Censo General de Población y Vivienda de 2010 el Instituto Nacional de Geografía e Informática de México cuantificó a la población china residente en México en 6,655 personas aproximadamente (INEGI, 2010:

${ }^{4}$ Varias instituciones políticas - mexicanas y chinas - no fueron consideradas por estas razones en la encuesta. 
17), equivalente a 0.7 por ciento de la población extranjera total en el país ${ }^{5}$ Sin embargo, según datos extraoficiales ofrecidos por Jimmy Li, secretario de la Confederación de Asociaciones Chinas en México (Cachimex), en 2009 dicha confederación estimó alrededor de 50,000 personas de origen chino, concentrados principalmente en el Distrito Federal, Chiapas y Mexicali. ${ }^{6}$

Reciente información proporcionada por el Instituto Nacional de Migración (INM, 2009-2013) —y por el momento sin posibilidad de lograr una serie histórica más larga - revela que más allá de su tamaño reducido, la población china en México se ha incrementado rápidamente en los últimos años: durante 2009-2013 el flujo migratorio total aumentó en más de 350 por ciento, alcanzando más de 12,000 ciudadanos chinos en 2013. Llama la atención, de igual forma, que la gran mayoría de los nacionales chinos aún ostente una calidad migratoria de relativamente corto plazo (como no inmigrante, prórrogas de esta misma calidad migratoria, así como de inmigrante; todas estas calidades con la posibilidad de alcanzar la calidad de inmigrado a través del cumplimiento de una serie de requisitos), mientras que los inmigrados apenas si representaron 2 por ciento del total del flujo migratorio en 2012.7 Esta reciente dinámica migratoria es significativa, después de prácticamente 50 años — desde 1920 hasta 1970 — con un descenso de la población china en el país (Xu, 2007).

Algunas de las primeras instituciones y asociaciones chinas en México de las que se tiene registro a finales del siglo xIX se encuentran en el estado de Sonora, donde se fundó la Asociación Mutualista Masónica o Logia Masónica

${ }^{5}$ El primer lugar lo ocupan residentes estadounidenses, con 76 por ciento de los extranjeros residentes en México.

${ }^{6}$ Quizá uno de los grupos extranjeros de mayor complejidad para censarlo y analizar su reproducción social y económica es el chino. Entre las distintas razones que explican esta dificultad destacan el hermetismo que guarda la comunidad china como una característica propia de su idiosincrasia, pero también por los antecedentes de racismo y xenofobia de la que fueron objeto en las primeras tres décadas del siglo xx, propiciados por el movimiento antichino que tuvo origen en Sonora y que se extendió al resto del país con diversas expresiones culminando en la masacre de varios cientos de ciudadanos chinos (Gómez, 1992).

7 El tema de las dificultades para obtener visas y una calidad migratoria tanto para turistas como inmigrantes chinos ha sido una queja constante por parte de todas las instituciones chinas en la última década (Oiu, 2014).

$116 \frac{\text { PRIMER SEMESTRE } 2016}{\text { MIGRACIÓN Y DESARROLLO NÚM. } 26}$ 
CUADRO 1

México, flujos migratorios de nacionales chinos (2009-2012)

\begin{tabular}{lcccrc}
\hline & 2009 & 2010 & 2011 & \multicolumn{1}{c}{2012} & 2013 \\
\hline No inmigrantes (FM3) & 666 & 1,778 & 2,416 & 1,984 & 1,548 \\
Prórrogas de no imigrantes & \multicolumn{1}{c}{ - } & \multicolumn{1}{c}{ - } & 4,292 & 4,988 & 5,663 \\
Inmigrante (FM2) & 1,958 & 1,736 & 1,144 & 807 & 4,743 \\
Refrendos de inmigrante & \multicolumn{1}{c}{-} & - & 5,953 & 5,970 & 106 \\
Inmigrados & 37 & 106 & 166 & 276 & - \\
Total & 2,661 & 3,620 & 13,971 & 14,025 & 12,060 \\
\hline
\end{tabular}

Taza de crecimiento

\begin{tabular}{llccrr}
\hline No inmigrantes (FM3) & - & 167.0 & 35.9 & -17.9 & -22.0 \\
Prórrogas de no imigrantes & - & - & - & 16.2 & 13.5 \\
Inmigrante (FM2) & - & -11.3 & -34.1 & -29.5 & 487.7 \\
Refrendos de inmigrante & - & - & - & 0.3 & -98.2 \\
Inmigrados & - & 186.5 & 56.6 & 66.3 & - \\
Total & - & 36.0 & 285.9 & 0.4 & -14.0 \\
\hline
\end{tabular}

Participación (porcentaje sobre el total)

\begin{tabular}{lccrrc}
\hline No inmigrantes (FM3) & 25.03 & 49.12 & 17.29 & 14.15 & 12.84 \\
Prórrogas de no imigrantes & \multicolumn{1}{c}{-} & - & 30.72 & 35.57 & 46.96 \\
Inmigrante (FM2) & 73.58 & 47.96 & 8.19 & 5.75 & 39.33 \\
Refrendos de inmigrante & - & - & 42.61 & 42.57 & 0.88 \\
Inmigrados & 1.39 & 2.93 & 1.19 & 1.97 & - \\
Total & 100.00 & 100.00 & 100.00 & 100.00 & 100.00 \\
\hline
\end{tabular}

Fuente: Elaboración propia con base en INM (2008-2013).

Chee Kung Tong (CHKT), siguiendo el modelo de asociación de distrito o huigan creada en Estados Unidos a mediados del mismo siglo con la finalidad de darle al inmigrante recién llegado apoyo económico o de algún otro tipo. Además, encontramos en el mismo estado una agrupación a modo de filial del Kuo Min Tang o Liga Nacionalista China, que entre sus filas tenía también a miembros de la CHKT. La presencia de la CHKT en Sonora fue resultado de las gestiones del entonces gobernador interino del estado, Ramón Corral, quien estableció contacto con la asociación en San Francisco entre 1887 y 1891 para reclutar a obreros y peones chinos en los puertos de Hong Kong y Shanghai para los centros de trabajo sonorenses (especialmente en las minas) 
(Cauich, 1998: 71). Con el tiempo dicha asociación estableció filiales en Nacozari, Empalme, Navojoa, La Colorada, Fronteras, Magdalena e Imuris; su estructura social fue consolidándose al punto de que entre sus miembros podían encontrarse banqueros, comerciantes, agricultores, oficinistas, jornaleros u obreros y, principalmente, comerciantes. Entre 1897 y 1910 la logia pasó de 10 afiliados a aproximadamente 2,000 socios en el estado. Para 1922, la logia contaba con un total de 5,000 socios, lo que equivaldría a 82.3 por ciento de la colonia china que vivía en Sonora a inicios de la década de 1920 (Cauich, 1998: 71-76).

Contemporáneamente existió la Unión Fraternal China, una sociedad encargada de proteger y velar por los chinos migrantes en toda la República Mexicana, que entre sus funciones incluía la tramitación de pasaportes y documentos consulares para que los chinos pudieran ingresar o salir del país libremente (Velásquez, 2005: 483). Paralelamente, en la primera década del siglo xx en Baja California Norte se estaba gestando un proceso en el que los migrantes chinos en la región comenzaron a participar como fuerza de trabajo en distintas actividades económicas: minería, agricultura, construcción del sistema ferroviario, etcétera. Teniendo como referencia las huigan sonorenses, se crea en 1914 la Logia Masónica China en Baja California, número 20, que será el parteaguas para la creación de la Asociación China de Mexicali hacia 1919, y también coexistía con una filial del Kuo Min Tang.

En otros estados de la República Mexicana se fue reproduciendo un esquema similar de organización de las comunidades de migrantes chinos siguiendo el mismo principio de ayuda y solidaridad. Ya hacia la tercera década del siglo xx, los estados de Chiapas, Chihuahua, Sinaloa, Tamaulipas, Yucatán, Veracruz y el Distrito Federal contaban con una presencia significativa de chinos, aunque no se puede concluir que llegaran a desarrollar niveles de asociación similares a los de Baja California Norte y Sonora.

Tanto las comunidades chinas que permanecieron en el país posterior al movimiento antichino como sus modos de asociación, experimentaron cambios en la cantidad y características de sus integrantes. La Revolución de 1949 y el establecimiento de relaciones diplomáticas entre México y China el 14 de febrero de 1972, marcaron un parteaguas en la composición de la 
inmigración china y sus instituciones. Desde entonces, la comunidad china ha estado principalmente integrada por distintas generaciones de mexicanos descendientes de migrantes chinos, chinos nacionalizados como mexicanos y residentes chinos. Es probablemente apenas a inicios del siglo XXI —como lo reflejan las estadísticas del cuadro 1-que encontramos una nueva generación de inmigrantes chinos en México.

En términos cualitativos, con base en la revisión bibliográfica, las múltiples entrevistas y la definición del universo de instituciones de inmigrantes chinos en México (véase cuadro 2) podemos en la actualidad clasificar a las asociaciones de inmigrantes chinos en México en cuatro grandes grupos: ${ }^{8}$

1. Instituciones históricas de inmigrantes chinos que surgieron en la primera mitad del siglo xx como resultado de expulsiones de fuerza de trabajo directas de China y/o de Estados Unidos, en el primer caso asociadas con diferencias políticas; muchas de estas corrientes iniciales provenían de Cantón. Como resultado, varias de estas instituciones están en sus orígenes vinculadas al Kuo Ming Tan y al anticomunismo y antimaoísmo. Existen, de igual forma, instituciones que en la segunda mitad del siglo xx se crearon para apoyar al gobierno central chino y el establecimiento de relaciones públicas — con miembros chinos y particularmente mexicanos-. Estas instituciones parecerían en la actualidad haber interrumpido su renovación generacional y entrado en crisis terminales, ante la menor relevancia de las discrepancias políticas sobre la República Popular China tanto en la propia China como en México y a nivel internacional.

2. Instituciones formadas por descendientes de inmigrantes chinos de hace varias generaciones que, por diferentes causas, han «descubierto» sus raíces chinas recientemente y buscan profundizar, difundir e incluso sacar provecho de este vínculo en su localidad, en México y en vínculos con China. Pasadas las condiciones antichinas que perduraron durante décadas del siglo Xx, en la actualidad chino-descendientes de diversas generaciones - jóvenes y no tanto-, en su mayoría de nacionalidad mexicana y sin mayor vínculo cultural,

${ }^{8}$ Con base en la definición de «instituciones de inmigrantes chinos» agrupaciones políticas mexicanas - algunas maoístas y activas a favor de las relaciones políticas con China - no son consideradas en esta agrupación, aunque relevantes en las instituciones generales entre México y China. 
lingüístico y de otra índole con China, buscan "conocer sus raíces» chinas, que en algunos casos datan de tres o cuatro generaciones. Estas instituciones en varios casos son informales y muy activas en los medios electrónicos.

Instituciones de inmigrantes chinos recientes con objetivos estrictamente comerciales: organismos, asociaciones y cámaras de empresarios con el objetivo específico de abordar temáticas puntuales en los negocios, con organismos más o menos cercanos a la Embajada de la República Popular China. Ante la muy importante presencia económica de China en México desde inicios del siglo xxi y el reciente establecimiento de empresas chinas (Dussel, 2014), estos organismos son importantes en la relación México-China y juegan un papel destacado en la organización y logística de eventos de la comunidad china en México. Destacan por su origen inmigrantes de Pekín, así como otras provincias chinas.

3. Instituciones de inmigrantes chinos resultado de la más reciente «oleada» de inmigrantes chinos (desde finales del siglo XX e inicios del siglo XXI): estudiantes, académicos, funcionarios de diversas instituciones chinas, funcionarios de instituciones como prensa y televisión, empleados de todos los niveles de las empresas chinas recientemente establecidas en México (Dussel, 2014), institutos de chino-mandarín, entre muchos otros. Esta nueva comunidad ingresa a México —apenas muy recientemente, incluso con sus respectivas familias - y se integra a instituciones de inmigrantes chinos en búsqueda de mantener sus lazos con China e integrarse en México, creando instituciones de orientación religiosa, ${ }^{9}$ cultural (deportivas y de caligrafía, etcétera). Por su origen, este grupo de inmigrantes es altamente heterogéneo, con individuos provenientes de buena parte de la zona costera de China (Shanghai, Hong Kong, Zhejiang, etcétera).

Esta nueva diversidad condiciona la estructura de cada asociación y define las relaciones entre sus miembros y con otras asociaciones. Al respecto, Mónica Cinco (1999) ofrece un breve y contundente análisis respecto a la forma en que los chinos migrantes de mediados del siglo XX, sus descendientes

9 Detectamos a un grupo de comunidades religiosas chinas dirigidas directamente a los «nuevos» inmigrantes chinos, aunque en general sin interés de establecer cualquier contacto y/o dar a conocer sus objetivos y experiencias recientes en México.

$120 \frac{\text { PRIMER SEMESTRE } 2016}{\text { MIGRACIÓN Y DESARROLLO NÚM. } 26}$ 
y las nuevas generaciones de chinos se relacionan y se asocian en la Ciudad de México, comportamiento que bien puede ser reproducido por otras asociaciones en distintas partes del país. La autora observa particularmente la inexistencia de una fuerte cohesión en el interior de los grupos de chinos migrantes y el grupo de descendientes de chinos. Ello se explica en buena medida por la diversidad de motivos que propiciaron su salida de China, lugares de procedencia, y fechas y contextos políticos de la migración. Marca una gran diferencia provenir de Cantón, Taiwán, Shanghai o Hong Kong, particularmente del periodo anterior a la conformación de la República Popular China, con respecto a los residentes chinos de finales del siglo xx y principios del xxi provenientes de otras regiones como Pekín. Ello explica que los migrantes cantoneses y taiwaneses, por ejemplo, no se sientan identificados con la China socialista que ayudó a fundar Mao Tse Tung, sino con la nacionalista de Sunt Yat-sen y posteriormente con Chian Kai-shek. Cinco resume lo anterior de la siguiente forma:

[...] la vida china en la Ciudad de México es la vida de personas y familias, no de una comunidad que en conjunto construya espacios y momentos para apoyarse, para reconocerse como iguales o simplemente para convivir. Basta mencionar que los eventos organizados por la Embajada China para festejar alguna fecha significativa para los chinos, son reuniones organizadas por y para chinos pequineses. Sólo un reducido número de chinos cantoneses que ha ascendido socialmente son invitados, mientras el resto de la población china que es mayoritariamente cantonesa, es excluida de todo aquello que tenga que ver con su embajada. Forman una periferia que en lo individual y al interior del grupo familiar celebran las fiestas que en conjunto los chinos mandarines festejan en la embajada. Pero tampoco los mandarines constituyen una comunidad solidaria (Cinco, 1999: 93). ${ }^{10}$

La heterogeneidad de los miembros de la comunidad china en México resulta en una red compleja y limitada que opera más en lo local que en lo

${ }^{10}$ El caso - la relación de la Embajada de China en México y su relación ante la creciente heterogeneidad de la comunidad china - sin lugar a dudas requiere de un mayor análisis hasta el 2015. 
nacional en sus vínculos con el exterior. Sólo aquellas instituciones vinculadas con el gobierno central chino y con sus grupos empresariales propician una comunicación constante con China y otros países como Estados Unidos. ${ }^{11}$

A lo largo de la República Mexicana se encontraron 61 asociaciones chinas con perfiles, miembros y actividades distintas. El grueso de estas organizaciones es de reciente creación y se concentra en actividades cívicoculturales y económicas, sin perder además sus objetivos de apoyo y solidaridad. Su estatus jurídico en la mayoría de los casos es informal al no estar registradas ante autoridad alguna, china o mexicana, que permita su identificación. El alcance de sus actividades en este sentido es limitado y se concentran en celebrar eventos modestos, como sucede en Coatzacoalcos (Veracruz) o en Puebla y Zacatecas, donde los grupos de descendientes chinos en años recientes se organizan para celebrar anualmente la fiesta del año chino. En las comunidades chinas de estas dos últimas entidades no se identificó la existencia de una «institución de inmigrantes chinos» según la definición inicial, aunque sí grupos que promueven y presenten danzas del león, del dragón y de la serpiente en celebraciones anuales.

En catorce estados del país se detectaron instituciones chinas: Baja California Norte y Sur, Chiapas, Chihuahua, Coahuila, Distrito Federal, Jalisco, Hidalgo, Nuevo León, Sinaloa, Sonora, Tamaulipas, Veracruz y Yucatán, siendo Baja California Norte y el Distrito Federal quienes cuentan con un mayor número de estas organizaciones con alrededor de 25 y 15 respectivamente (véase cuadro 2 ). ${ }^{12}$

${ }^{11}$ Estas tendencias también se manifiestan incluso en organismos empresariales en la actualidad. En varios casos se constataron fuertes tensiones entre grupos empresariales cercanos y con mayor independencia - en varios casos también resultado de que varios de sus miembros ya tienen una residencia permanente en México o incluso la nacionalidad mexicana, así como una larga trayectoria y experiencias en México- a la Embajada de la República Popular China en México y en eventos específicos como el desfile de la comunidad china en la Ciudad de México (2009-2011) y la ExpoChina-México realizadas también en la Ciudad de México en 2009-2011. Estas tensiones también implicaron diversas dificultades por parte de las contrapartes mexicanas y fueron factores importantes para comprender su término.

${ }^{12}$ Es importante reconocer que en el futuro habría que revisar y contactar a cada una de estas instituciones de comunidades chinas. Como se verá para el caso donde se realizó trabajo de

$122 \frac{\text { PRIMER SEMESTRE } 2016}{\text { MIGRACIÓN Y DESARROLLO NÚM. } 26}$ 


\section{CUADRO 2}

Universo de instituciones de inmigrantes chinos en México

\begin{tabular}{|c|c|c|}
\hline MUNICIPIO & ASOCIACIÓN & PRINCIPAL ACTIVIDAD \\
\hline \multicolumn{3}{|c|}{ BAJA CALIFORNIA NORTE } \\
\hline \multirow[t]{25}{*}{ Mexicali } & Asociación de Mexicali & $\begin{array}{l}\text { Cultural y representar a la } \\
\text { comunidad china ante cual- } \\
\text { quier instancia gubernamen- } \\
\text { tal y civil. }\end{array}$ \\
\hline & Asociación Zhong Shan & Cívico-culturales \\
\hline & Asociación Lung Kung & Cívico-culturales \\
\hline & Escuela de Idioma ChinoTianji & Enseñanza del mandarín \\
\hline & $\begin{array}{l}\text { Logia Masónica China en Baja California, } \\
\text { Núm. } 20 \text { (Ming ChihTang) }\end{array}$ & \\
\hline & Asociación Chung Shan de Baja California & Cívico-culturales \\
\hline & Asociación Sam Yap A.C. & Cívico-culturales \\
\hline & Asociación Leon Chong How Tog & Cívico-culturales \\
\hline & Asociación Hoy Yin & Cívico-culturales \\
\hline & Asociación Chew Lun & Cívico-culturales \\
\hline & Asociación Yee Fong Toy Tong & Cívico-culturales \\
\hline & Asociación Sui Yue Tong & Cívico-culturales \\
\hline & Asociación Hu Suy Shan Tong & Cívico-culturales \\
\hline & Asociación Wong Kong Har Tong & Cívico-culturales \\
\hline & Asociación Wong Wu Sun & Cívico-culturales \\
\hline & Asociación Nam Ping & Cívico-culturales \\
\hline & Asociación Long Kong & Cívico-culturales \\
\hline & Asociación Lon Sai & Cívico-culturales \\
\hline & Asociación Gee How Oak Tin & Cívico-culturales \\
\hline & Asociación Lun Tack Tong & Cívico-culturales \\
\hline & Asociación Chi Tak Tong & Cívico-culturales \\
\hline & Escuela China de Mexicali & Educativa \\
\hline & Iglesia Bautista China de Mexicali & Religiosa \\
\hline & Cámara de Empresarios Chinos de México A.C. & Económica \\
\hline & $\begin{array}{l}\text { Centro de la Investigación de la Cultura China } \\
\text { de Baja California }\end{array}$ & Educativo-cultural \\
\hline \multirow[t]{2}{*}{ Tijuana } & Comunidad China en Tijuana & Cívico-culturales \\
\hline & Asociación de la Colonia China en Tijuana & Cívico-culturales \\
\hline
\end{tabular}

campo, muchas de estas instituciones no muestran actividades específicas durante largos periodos. 


\section{CUADRO 2 (Continuación)}

\begin{tabular}{|c|c|c|}
\hline MUNICIPIO & ASOCIACIÓN & PRINCIPAL ACTIVIDAD \\
\hline & $\begin{array}{l}\text { Instituto Confusio de la Ciudad de México } \\
\text { Huaxia }\end{array}$ & Enseñanza del Mandrín \\
\hline \multicolumn{3}{|c|}{ GUADALAJARA } \\
\hline Jalisco & $\begin{array}{l}\text { Casa de la Cultura China } \\
\text { Comunidad China del Águila y el Dragón, A.C. } \\
\text { (Long Yin) }\end{array}$ & $\begin{array}{l}\text { Cultura } \\
\text { Cívico-culturales }\end{array}$ \\
\hline \multicolumn{3}{|c|}{ HIDALGO } \\
\hline Pachuca & Comunidad China en Hidalgo, A.C. & Cívico-culturales \\
\hline \multicolumn{3}{|c|}{ NUEVO LEÓN } \\
\hline Monterrey & $\begin{array}{l}\text { Instituto Confusio de la Universidad Autóno- } \\
\text { ma de Nuevo León }\end{array}$ & Enseñanza del Mandarín \\
\hline \multicolumn{3}{|c|}{ PUEBLA } \\
\hline & Comunidad China en Puebla & Cívico-culturales \\
\hline \multicolumn{3}{|c|}{ SONORA } \\
\hline & Asociación de la Colonia China de Sonora & Cívico-culturales \\
\hline \multicolumn{3}{|c|}{ SINALOA } \\
\hline Los Mochis & Comunidad Asiática de los Mochis & \\
\hline \multicolumn{3}{|c|}{ TAMAULIPAS } \\
\hline Tampico & Comunidad China de Tampico, A.C. & Cívico-culturales \\
\hline \multicolumn{3}{|c|}{ VERACRUZ } \\
\hline Coatzacoalcos & Comunidad China de Coatzacoalcos, A.C. & $\begin{array}{l}\text { Promoción cultural y de la } \\
\text { medicina tradicional china }\end{array}$ \\
\hline \multicolumn{3}{|c|}{ YUCATÁN } \\
\hline Mérida & $\begin{array}{l}\text { Asociación China Península de Yucatán, México } \\
\text { Instituto Confusio de la Universidad Autónoma } \\
\text { de Yucatán }\end{array}$ & $\begin{array}{l}\text { Cívico-culturales } \\
\text { Enseñanza del Mandarín }\end{array}$ \\
\hline
\end{tabular}

Fuente: Elaboración propia. 


\section{Principales Resultados de las ASOCIACIONES CHINAS ENTREVISTADAS ${ }^{13}$}

En total se entrevistaron a 12 instituciones de inmigrantes chinos en tres áreas, consideradas de las más representativas e ilustrativas de la realidad y asimetrías que enfrenta la comunidad china y su sistema organizacional.

- Baja California Norte (Mexicali): Asociación China de Mexicali (fecha de creación: 1919), Asociación Zhong Shan (1915), Asociación Lung Kung (1920), Escuela de Idioma Chino Tianji (2013).

- Chiapas (Tapachula y Escuintla): Comunidad China del Soconusco (1983), Grupo Danzante Artístico de Dragón y Leones Chino (1987), Comunidad Escuintleca de China (2009), Agrupación de Restauranteros Chinos de Tapachula (en formación).

- Distrito Federal: Fundación Cultural China A.C. (1973), Consejo Chino para el Fomento del Comercio Internacional (1988), Asociación de Empresarios Zhonghua en México/Cámara de Empresarios Zhenjia en México (2010), Grupo Inmigraciones Chinas a México (2012) y Grupo Cultural de Danzas Chinas (2013).

Adicionalmente se pudo obtener información cualitativa valiosa de otras fuentes directas que permitieron comprender desde otra perspectiva y con mayor profundidad la dinámica de la comunidad y asociaciones chinas en las entidades visitadas. ${ }^{14}$ Lo anterior, sumado a lo relatado por los actores centrales de la investigación, nos permite resumir lo siguiente:

${ }^{13}$ Estamos muy agradecidos por el apoyo ofrecido por un grupo de personas e instituciones para realizar el trabajo durante el proyecto, particularmente por parte de Esteban León de la Asociación China de Mexicali, Alma Delia Fuentes Chié, María Magdalena Chiang y Adolfo Joo Trujillo de la Asociación China del Soconusco A.C. y Mónica Cinco Basurto, académica y entusiasta organizadora de diversos eventos sobre la diáspora china en México.

${ }^{14}$ Merece resaltar en este caso al Instituto de Investigaciones Históricas de la Universidad Autonóma de Baja California, a través de la doctora Maricela González y el doctor Servado Ortoll; el Departamento de Planeación y Desarrollo Municipal del Municipio de Tapachula-Chiapas a través del licenciado José Antonio Sánchez y la maestra Mónica Cinco Basurto en el Distrito Federal.

$126 \frac{\text { PRIMER SEMESTRE } 2016}{\text { MIGRACIÓN Y DESARROLLO NÚM. } 26}$ 
1. En Mexicali se encuentran cuatro de las asociaciones de mayor antigüedad en México. La mayoría de los miembros del total de las organizaciones en el área son actualmente descendientes de segunda y hasta cuarta generación de las distintas migraciones chinas provenientes principalmente de Cantón, y de nacionalidad mexicana. En menor proporción se encuentran chinos nacionalizados mexicanos y residentes chinos. Entre sus principales objetivos y actividades están las de carácter cívico-cultural, económicas. Algunas de ellas brindan apoyo migratorio a chinos recién llegados, como es el caso de la Asociación China de Mexicali, que a su vez funciona como una federación que reúne asociaciones más pequeñas, la Asociación Zhong Shan y la Asociación Lung Kung. Su vinculación con las autoridades locales mexicanas básicamente tiene lugar a través de actividades de carácter cultural como el del año nuevo chino y algunos otros eventos como exposiciones de pintura, danzas y gastronomía china.

A decir de Esteban León, vicepresidente de la Asociación China de Mexicali, la red que conforman con sus asociaciones agremiadas (cuenta con 500 miembros aproximadamente) es sólida a nivel regional más que nacional y la comunicación directa con China es limitada. Por el contrario, la Cámara de Empresarios Chinos de México, integrada por distintos tipos de miembros (chinos naturalizados mexicanos, residentes y descendientes de migrantes chinos) de Baja California Norte y Sur, Sonora y Chihuahua, tiene una proyección regional y está construyendo sistemáticamente relaciones más estrechas con sus contrapartes en China y en Estados Unidos.

2. En Chiapas las asociaciones chinas son de muy reciente creación y presentan un menor nivel de integración recíproca para realizar proyectos conjuntos, lo que a su vez determina que su vinculación con el gobierno local o estatal sea limitada, y con el extranjero (y China) prácticamente nula. Sus miembros son en su totalidad mexicanos descendientes de varias generaciones de chinos provenientes principalmente de Cantón. En Tapachula se encuentra la asociación más representativa y formalmente instituida, la Comunidad China del Soconusco A.C., que data del año 1983 y en 2014 adquirió el carácter de asociación civil. Se pueden encontrar asociaciones similares en los municipios de Escuintla, Huixtla y Mazatlán, todas ellas operando de manera informal y sin estar constituidas legalmente. 
Varios miembros de la comunidad china de la región coincidieron en que el obstáculo mayor para mantener viva la identidad china entre las nuevas generaciones de descendientes chinos y recientes migrantes es la falta de recursos económicos y cohesión entre la comunidad, que impide impulsar proyectos que vayan más allá de la simple exposición de danzas y la celebración del Año Nuevo Chino. A ello se suma la falta de reconocimiento del gobierno estatal y local, e incluso de la propia Embajada de la República Popular China en México. En este sentido, la Comunidad China del Soconusco A.C. pretende posicionarse como la "punta de lanza» para trascender al igual que lo han hecho otras asociaciones en el país. ${ }^{15}$ Quizá la situación pueda transformarse a partir del próximo convenio de hermanamiento entre Tapachula y la ciudad de Dongying en China, con el que se busca impulsar el intercambio comercial, cultural, social, económico y turístico y pudiera crear nuevas condiciones para la propia institución.

3. Las asociaciones chinas en el Distrito Federal presentan una mayor diversidad e interacción recíproca, una mayor vinculación con la sociedad mexicana y las autoridades locales y federal, así como con el gobierno central y otras instituciones públicas chinas. Entre sus miembros se cuentan descendientes de migrantes chinos, chinos nacionalizados mexicanos y residentes. El impacto de la «nueva oleada» de inmigrantes chinos y turistas en la Ciudad de México ha sido importante, aunque todavía no ha sido analizada ni cuantificada en detalle. ${ }^{16}$ Las asociaciones son de carácter cívico-cultural, religiosas, económicas y de enseñanza del idioma chino. A diferencia de las áreas previamente mencionadas, en el Distrito Federal encontramos asociaciones e

${ }^{15}$ Existen otros actores que a través de iniciativas individuales se esmeran en tratar de mantener viva la herencia de sus antepasados como es el caso del director del Grupo Danzante Artístico de Dragón y Leones Chino, Julio Pui Chong, una de las agrupaciones más representativas de danza artística y del ingeniero Alejandro Juan Chan, propietario de uno de los restaurantes chinos más antiguos del Soconusco. Este último impulsa una agrupación de restauranteros chinos de la zona.

${ }^{16}$ Nos referimos puntualmente a los empleados de empresas chinas que recientemente han invertido en México, así como de cientos de estudiantes, académicos e investigadores que actualmente han ingresado —en su mayoría en forma temporal- en México; tan sólo el Centro de Enseñanza para Extranjeros (CEPE) de la UNAM integra bimestralmente a más de 200 estudiantes chinos aprendiendo español en sus diferentes niveles, mientras que empresas como Huawei actualmente emplean a más de 1,600 personas en la Ciudad de México (Dussel, 2014) y con una participación de nacionales chinos importante.

$128 \frac{\text { PRIMER SEMESTRE } 2016}{\text { MIGRACIÓN Y DESARROLLO NÚM. } 26}$ 
instituciones chinas que buscan mejorar la relación entre ambos países. Ejemplo de ellas son la Asociación de Empresarios Zhonghua en México/Cámara de Empresarios Zhenjia en México, el Consejo Chino para el Fomento del Comercio Internacional, la Cámara de Representación Empresarial China en México y el Instituto "Confucio» (que cuenta con cinco representaciones en México y dos en el Distrito Federal).

En las tres áreas hay migrantes chinos bajo diversos estatus legales de residencia, aunque éstos no están correlacionados con el tipo de organización. Por otra parte, la realidad económica, política y social de cada estado se impone para definir la dinámica de la respectiva comunidad china. En definitiva, de los tres casos revisados en el estado de Chiapas la comunidad china y sus asociaciones presentan mayores problemas de integración y vinculación a nivel local, estatal, nacional e internacional. Sólo algunos miembros sostienen a título individual comunicación constante con el extranjero, más por motivos personales que para beneficiar a la comunidad.

\section{Características generales}

A continuación se exponen los resultados más significativos de la encuesta (véase cuadro 3).

En primer lugar, 80 por ciento de sus miembros fundadores proceden de la provincia de Guangdong (Cantón), 10 por ciento de Zhejiang y 10 por ciento de otras provincias. En cuanto a su origen, 42 por ciento de éstas se fundaron entre 2000 y 2013; 33 por ciento entre 1970 y 1999 y 25 por ciento entre 1915 y 1920 . En cuanto a los principales objetivos de las instituciones de inmigrantes chinos, 50 por ciento son culturales y cívicas, 25 por ciento busca fines económicos-comerciales y 25 por ciento apoyar a migrantes chinos. 


\section{CUADRO 3}

Características generales de las asociaciones chinas en México (2014) (en porcentaje)

\begin{tabular}{|c|c|}
\hline CONCEPTO & $\%$ \\
\hline \multicolumn{2}{|l|}{ PROVINCIA DE PROCEDENCIA } \\
\hline Guangdong (Cantón) & 80 \\
\hline Zhejiang & 10 \\
\hline Pekín & 10 \\
\hline \multicolumn{2}{|l|}{ TIPO DE ORGANIZACIÓN } \\
\hline Cultural & 50 \\
\hline Económica & 25 \\
\hline Apoyo a migrantes & 25 \\
\hline \multicolumn{2}{|l|}{ AÑO DE CREACIÓN } \\
\hline $1915-1920$ & 25 \\
\hline $1970-1999$ & 33 \\
\hline $2000-2013$ & 42 \\
\hline \multicolumn{2}{|l|}{ Estatus Legal } \\
\hline Opera informalmente & 17 \\
\hline Registrada legalmente en México como organización sin ánimo de lucro & 33 \\
\hline $\begin{array}{l}\text { Registrada legalmente en México como organización sin ánimo de lucro y } \\
\text { cuenta con un código de excepción de impuestos }\end{array}$ & 33 \\
\hline Está registrada en la Embajada o Consulado de China & 17 \\
\hline \multicolumn{2}{|l|}{ LUGAR DONDE REALIZA SUS ACTIVIDADES } \\
\hline Exclusivamente en México & 58 \\
\hline Principalmente en México & 17 \\
\hline En ambos países & 25 \\
\hline \multicolumn{2}{|l|}{ TIENE RELACIONES REGULARES CON CHINA } \\
\hline Sí & 58 \\
\hline No & 42 \\
\hline \multicolumn{2}{|l|}{ PRESUPUESTO MENSUAL } \\
\hline No tiene gastos mensuales & 42 \\
\hline Menos de MX $\$ 5^{\prime} 000$ & 8 \\
\hline Menos de MX $\$ 10^{\prime} 000$ & 2 \\
\hline Menos de MX $\$ 30^{\prime} 000$ & 25 \\
\hline Menos de MX $\$ 50 ’ 000$ & 8 \\
\hline Otro & 15 \\
\hline
\end{tabular}


En segundo lugar, es interesante que 33 por ciento de las asociaciones están registradas legalmente en México como organizaciones sin ánimo de lucro y cuentan con un código de excepción de impuestos; sólo 17 por ciento están registradas ante la embajada de China en México, operando el resto informalmente. Respecto al lugar donde realizan sus actividades y operaciones, 58 por ciento lo hace sólo en México, 25 por ciento en México y China y 25 por ciento principalmente en México. ${ }^{17}$ Como resultado, sólo 58 por ciento de las instituciones encuestadas tienen relaciones con China con cierta periodicidad.

Tercero, importa notar que 42 por ciento de las instituciones no cuenta con ingreso mensual alguno; 8 por ciento cuenta con recursos iguales o inferiores a los 50,000 pesos mensuales.

Características generales de los miembros de las instituciones de inmigrantes chinos

Entre las asociaciones chinas entrevistadas se encontró que 51 por ciento de sus miembros son hombres y 49 por ciento mujeres. De igual forma, 81 por ciento de los miembros tiene entre 25-55 años, representando los mayores de 55 años apenas 6 por ciento de los miembros de las instituciones encuestadas.

En cuanto a su nivel de escolaridad, 86 por ciento tiene estudios de preparatoria o inferiores; existen sin embargo grandes disparidades según la institución específica: en dos casos, por ejemplo, la totalidad de los miembros tenía estudios universitarios o de posgrado. Entre las ocupaciones de los miembros se reportó que 33 por ciento es dueño de negocios o empresas; 29 por ciento se compone de profesionistas o técnicos; 24 por ciento de estudiantes activos a diversos niveles educativos, y 14 por ciento de empleados de oficina o vendedores.

${ }_{17}$ Este rubro se refiere a que son asociaciones chinas que fueron creadas por el gobierno central de China para operar en México, tal es el caso del cCPIT. 
Respecto a su estatus legal, 71 por ciento de los miembros de las instituciones encuestadas son ciudadanos mexicanos, 9 por ciento no tiene papeles o visa que acrediten su estancia en México y 9 por ciento tiene un permiso temporal de estancia. De la mayor relevancia es que todos los miembros de las instituciones encuestadas cuenten con más de cinco años de estancia en México y 73 por ciento con más de 10 años. Esto sugiere o bien un sesgo de la muestra hacia este universo de chinos residentes en México, o bien que sólo los chinos con una residencia más larga en México participan en las instituciones de inmigrantes chinos. El tema es de la mayor relevancia y debiera profundizarse en el futuro mediante encuestas y entrevistas en profundidad para comprender la más reciente inmigración china. ${ }^{18}$

Por último, de las 12 asociaciones chinas y sus miembros entrevistados, 33 por ciento reportaron que se comunican con China por cuestiones familiares o de negocios; 25 por ciento lo hace una vez al año o menos, 8 por ciento al menos dos veces al mes y 33 por ciento nunca o rara vez tienen comunicación. Por otra parte, estas instituciones de inmigrantes chinos en México establecen contacto con instituciones públicas o privadas en China al menos una vez al año en 75 por ciento y una vez a la semana 25 por ciento de los casos. El 50 por ciento de los miembros de las instituciones viaja a China al menos una vez al año, mientras que el restante 50 por ciento nunca o rara vez lo hace. El 67 por ciento de las instituciones, de igual forma, indicó que nunca o rara vez envía representantes a China por asuntos vinculados con su institución. En la mayoría de los casos estos contactos de los miembros de las asociaciones chinas son de carácter personal y no para desarrollar actividades de las respectivas instituciones.

${ }^{18}$ En forma de hipótesis pudiera plantearse que la más reciente inmigración china está acompañada por esfuerzos de las respectivas instituciones receptoras como pudieran ser sus empresas (chinas u otras), universidades u otras instituciones. Existe también un ámbito informal/ilegal de nacionales chinos que, por el momento, no se ha investigado suficientemente, así como sus respectivas instituciones de inmigrantes.

$132 \frac{\text { PRIMER SEMESTRE } 2016}{\text { MIGRACIÓN Y DESARROLLO NÚM. } 26}$ 


\section{CUADRO 4}

Características de los miembros de las instituciones de inmigrantes chinos

(porcentaje)

\begin{tabular}{|c|c|}
\hline $\mathrm{EDAD}$ & $\%$ \\
\hline Menor de 25 & 11 \\
\hline De 25 a menos de 40 & 48 \\
\hline De 40 a menos de 55 & 33 \\
\hline Mayores de 55 & 33 \\
\hline No indicó & 2 \\
\hline Promedio de mujeres & 49 \\
\hline Promedio de hombres & 51 \\
\hline \multicolumn{2}{|c|}{ NIVEL DE EDUCACIÓN } \\
\hline Primaria & 2 \\
\hline Secundaria & 37 \\
\hline Preparatoria & 44 \\
\hline Universidad o más & 11 \\
\hline No indicó & 3 \\
\hline \multicolumn{2}{|c|}{ OCUPACIÓN DE LOS MIEMBROS } \\
\hline Empleados de oficina o vendedores & 14 \\
\hline Profesionales o técnicos & 29 \\
\hline Dueños de negocios o empresas & 33 \\
\hline Estudiantes & 24 \\
\hline \multicolumn{2}{|c|}{ AÑOS DE ESTANCIA EN MÉXICO } \\
\hline Menos de 5 años & 0 \\
\hline De 5 a 10 años & 26 \\
\hline Más de 10 años & 12 \\
\hline Toda su vida & 61 \\
\hline \multicolumn{2}{|c|}{ ESTATUS LEGAL } \\
\hline No tienen papeles o visa & 9 \\
\hline Tienen permiso temporal & 9 \\
\hline Son residentes & 3 \\
\hline Son ciudadanos mexicanos & 71 \\
\hline No indicó & 8 \\
\hline
\end{tabular}




\begin{tabular}{|c|c|}
\hline FRECUENCIA DE COMUNICACIÓN CON CHINA & $\%$ \\
\hline Una vez a la semana & 33.3 \\
\hline $\mathrm{Al}$ menos dos veces al mes & 8.3 \\
\hline $\mathrm{Al}$ menos una vez al año o menos & 25 \\
\hline Nunca o rara vez & 33.3 \\
\hline \multicolumn{2}{|c|}{$\begin{array}{l}\text { FRECUENCIA CON LA QUE INSTITUCIONES CHINAS ESTABLECEN CONTACTO } \\
\text { CON LA ORGANIZACIÓN }\end{array}$} \\
\hline Una vez a la semana & 25 \\
\hline $\mathrm{Al}$ menos dos veces al mes & 17 \\
\hline Al menos una vez al año o menos & 33 \\
\hline Nunca o rara vez & 25 \\
\hline \multicolumn{2}{|c|}{$\begin{array}{l}\text { NÚMERO DE VECES AL AÑO QUE VIAJAN LOS MIEMBROS APROVECHANDO } \\
\text { VIAJES FAMILIARES O DE NEGOCIOS PARA TRATAR ASUNTOS RELACIONADOS } \\
\text { CON LA ORGANIZACIÓN }\end{array}$} \\
\hline $\mathrm{Al}$ menos un viaje al año & 50 \\
\hline Nunca o rara vez & 50 \\
\hline \multicolumn{2}{|c|}{$\begin{array}{l}\text { NÚMERO DE VECES AL AÑO QUE VIAJAN LOS MIEMBROS EXCLUSIVAMENTE } \\
\text { PARA TRATAR ASUNTOS RELACIONADOS CON LA ORGANIZACIÓN }\end{array}$} \\
\hline Al menos un viaje al año & 25 \\
\hline Nunca o rara vez & 67 \\
\hline
\end{tabular}

Fuente: Elaboración propia.

\section{Conclusiones}

Este documento contribuye al creciente conocimiento sobre las «instituciones transnacionales» $y$ en particular al caso de las instituciones de inmigrantes chinos en América Latina y el Caribe. La información y antecedentes sobre la comunidad china en México es por el momento muy limitada y requerirá en el futuro de mucha mayor atención y profundidad. El estudio encontró importantes limitaciones estadísticas y el trabajo de campo chocó con barreras importantes para abarcar una muestra mayor de las instituciones de inmigrantes chinos en México. No obstante lo anterior, la investigación arroja resultados significativos y sugiere hipótesis a profundizar para el futuro.

$134 \frac{\text { PRIMER SEMESTRE } 2016}{\text { MIGRACIÓN Y DESARROLLO NÚM. } 26}$ 
En general, por razones históricas en México y políticas en China, la comunidad china en México y sus instituciones de inmigrantes son reducidas e incomparables en términos de tamaño con las de otros países como Estados Unidos y Perú. Dificultades pasadas y actuales para el otorgamiento de visas a la población en China, ${ }^{19}$ las reminiscencias culturales — racistas y de otra índole - del movimiento anti-chino de las primeras décadas del siglo xx, y una generalizada percepción de China como «amenaza» y actitud "crítica» por parte de ciertos sectores sociales explican parcialmente este fenómeno. No obstante, cambios en la relación entre México y China, y la significativa presencia china a nivel global y en México en los ámbitos económico y político, han generado un aumento muy importante de la inmigración china desde finales del siglo xx e inicios del siglo XxI: tan sólo durante 2009-2013 los flujos migratorios de nacionales chinos a México aumentaron en más de un 350 por ciento, reconociendo todavía una base limitada.

A título de hipótesis a profundizar y contrastar en el futuro fueron identificadas en el presente al menos cuatro agrupaciones de instituciones de inmigrantes chinos en México. En primer lugar, las «históricas», resultantes de flujos migratorios desde Estados Unidos y directamente desde China en respuesta a la demanda de fuerza de trabajo barata y, en el segundo caso, de disidencias políticas. A ellas se suma un importante crecimiento de otros tres tipos de agrupaciones, resultado de los cambios más recientes en la relación México-China y la internacionalización de la propia China: instituciones de inmigrantes chinos de varias generaciones (en su mayoría de nacionalidad mexicana) en búsqueda de sus «raíces» chinas, instituciones de inmigrantes chinos con fines económico-comerciales, e instituciones vinculadas a la muy reciente "oleada» de inmigrantes chinos desde la primera parte del siglo XXI que reclutan sus miembros más allá del ámbito empresarial: estudiantes, académicos, médicos, funcionarios de diversas instituciones

${ }^{19}$ La empresa Huawei, por ejemplo, incluso en 2015 canceló varias de sus actividades en México para trasladarlas a Panamá, afectando a más de 500 empleos en México. La principal razón señalada por la propia empresa para la decisión (Dussel, 2014) fue la dificultad en obtener visas para realizar sus actividades adecuadamente. 
chinas y maestros, entre otros. Estas últimas instituciones tienen relaciones más estrechas con la República Popular China, y buscan objetivos religiosos, culturales, deportivos y culturales con China y entre China y México.

Algunas de estas nuevas instituciones se vinculan directamente con contrapartes institucionales o personales en China, en algunos casos creados por el gobierno central chino y en estrecha comunicación con la embajada, mientras que otras exhiben mayor independencia y hasta tensiones con los respectivos funcionarios responsables. Tal es el caso, por ejemplo, de un grupo de actividades y reuniones periódicas convocadas por la propia embajada y el CCPIT, ya sea en el ámbito empresarial-comercial y/o para conmemorar el Año Nuevo Chino, entre otros. Las nuevas asociaciones chinas que constituyen la «nueva oleada» de inmigrantes rebasan, sin embargo, a estas actividades: en algunos casos en estrecha colaboración con la embajada (como en el caso de exposiciones comerciales y desfiles), pero en otros casos como de asociaciones religiosas, de caligrafía, deporte y de actividades culturales, educativas y académicas, estas últimas reflejan una creciente independencia de la embajada.

Esas nuevas asociaciones buscan diversos objetivos entre los que destacan el mejoramiento de la percepción que se tiene de China en México, la divulgación de la cultura y el lenguaje, y la generación de condiciones adecuadas para las empresas y empresarios chinos en México. Como resultado, si bien estas asociaciones coexisten en todo el territorio mexicano, no todas gozan del reconocimiento y apoyo de la embajada, ya sea por la heterogeneidad de sus actividades y/o porque están integradas por chinos nacionalizados como mexicanos y mexicanos descendientes de chinos. Por el momento la embajada no está incluso vinculada y/o tiene información de estas asociaciones y sus respectivas actividades, aunque al parecer tiene un gran interés y apertura a integrarse — deberá definir en el futuro formas específicas-a las mismas.

La revisión bibliográfica, el estudio de campo y la encuesta responden parcialmente a los cuatro tipos de instituciones arriba señalados. En Mexicali y la Ciudad de México se aprecian claramente los cuatro tipos de instituciones, desde las de carácter más político y las «tradicionales» con varias 
décadas de existencia, hasta otras de muy reciente creación y con fines culturales más profundos y vínculos cotidianos. En el Distrito Federal confluyen todas estas instituciones de inmigrantes, tanto por la centralización política y económica de México, como por la existencia de la Embajada de la República Popular China y el establecimiento de los principales centros culturales y económicos de chinos en México. La centralización de los centros educativos y del fomento económico a la relación México-China también contribuye a esta concentración. Los casos de Mexicali y Chiapas, por otro lado, reflejan casos diferentes de la migración china a México. Mientras que en Mexicali se encuentra la más añeja comunidad china en México, el impacto de la «nueva oleada» de inmigrantes chinos ha sido todavía más limitado, aunque con señaladas excepciones.

En Chiapas y las diversas comunidades y municipios analizados allí, las instituciones existentes datan desde la década de los ochenta del siglo xx y todavía en cantidades y con grados de integración más limitados que en Mexicali y el Distrito Federal. En este caso, la gran mayoría de las instituciones chinas cuentan tanto con miembros chinos de varias generaciones como con otros de nacionalidad mexicana; aspiran a beneficiarse con la recuperación y representación de estos lazos comunes entre los miembros ante autoridades locales, regionales, nacionales y hasta en China. ${ }^{20}$ Ejemplo de ello es la Asociación China del Soconusco, A.C. en Chiapas que recientemente adquirió su reconocimiento legal como asociación civil. En la entrevista, los dirigentes ${ }^{21}$ de dicha asociación mencionaron que entre los objetivos que persiguen se cuenta el de alcanzar un mayor reconocimiento en México y con China para poder atraer recursos financieros y fomentar la cultura china entre los jóvenes y paralelamente emprender algún tipo de proyecto productivo.

Las estadísticas, el trabajo de campo y la encuesta también sugieren que la más reciente inmigración china pareciera todavía no integrarse directamente

${ }^{20}$ Es decir, los miembros de las asociaciones esperan en algunos casos que al destacar ser miembro de la comunidad china-mexicana pudieran obtener recursos — por ejemplo de instituciones mexicanas y/o chinas - para el fomento de las actividades culturales y comerciales entre sus respectivas localidades y con China.

${ }^{21}$ Alma Delia Fuentes Chié, María Magdalena Chiang y Adolfo Joo Trujillo. 
a las instituciones de inmigrantes $-\mathrm{y}$, como contraparte, que las asociaciones chinas analizadas están conformadas en su mayoría por mexicanos descendientes de diferentes generaciones de migrantes chinos- En menor proporción hay asociaciones formadas por chinos nacionalizados mexicanos y residentes de la República Popular China. Dicha heterogeneidad, como se mencionó, dificulta una mayor cohesión entre los migrantes recién llegados y los descendientes de dos o tres generaciones atrás. Entre los factores explicativos de este desempeño también es relevante señalar que los descendientes en su mayoría ya no hablan chino e incluso ya no son considerados chinos por la inmigración china más reciente; el «diálogo» entre estas diversas generaciones de inmigrantes chinos es entonces complejo y no se encontró una institución que procure facilitar la comunicación intergeneracional.

Entre las principales actividades que realizan se cuentan aquellas de carácter cívico-cultural para preservar y difundir las tradiciones y cultura china; otras se especializan en promover la enseñanza del mandarín y facilitar las operaciones económico-comerciales de las empresas con capital chino y sino-mexicano. Sólo algunas aún promueven la defensoría y apoyo legal migratorio y económico para aquellos chinos que buscan establecerse en México o en Estados Unidos.

Respecto al nivel de integración y desarrollo de redes que va de lo local a lo nacional e internacional, sólo algunas tienen como objetivo este tipo de funciones (por ejemplo las cámaras o asociaciones de empresarios registradas en México o para la enseñanza del mandarín como el Instituto «Confucio»), organizando eventos significativos como los señalados desfiles y la Expo China-México en la Ciudad de México.22 El resto de las asociaciones tiene comunicación y vinculación limitadas y en algunos casos nulas, establecidas en algunos casos a nivel individual más que organizacional. La Expo

22 La Expo China-México, por ejemplo, se realizó durante 2009-2011 bajo el liderazgo de la Confederación de Asociaciones Chinas en México (Cachimex) y la Cámara General de Empresarios Chinos en México, conjuntamente con la Confederación Patronal de la República Mexicana (Coparmex), el Instituto Nacional para el Federalismo y el Desarrollo, Secretaría de Gobernación (Inafed) y el Cechimex (UNAM). En diversos eventos participaron adicionalmente múltiples empresarios y organismos empresariales mexicanos y chinos.

$138 \frac{\text { PRIMER SEMESTRE } 2016}{\text { MIGRACIÓN Y DESARROLLO NÚM. } 26}$ 
China-México en sus diversas ediciones vinculó a las pequeñas y medianas empresas mexicanas a gobiernos estatales y municipales para presentar propuestas ejecutivas ante el Banco de Desarrollo de China y con otras empresas chinas.

Nos parece que flujos migratorios e instituciones de inmigrantes chinos crecerán en el corto y mediano plazo. Tanto las instituciones con miembros de nacionalidad mexicana como aquellas con objetivos económico-comerciales, así como la heterogénea inmigración reciente de estudiantes, funcionarios, empresarios y empleados chinos con certeza buscarán nuevas vías para integrarse temporalmente o por largos periodos en México.

Por último, la investigación destaca un grupo de factores para comprender la diferente conformación de la comunidad china en México y sus respectivas instituciones:

1. Las condiciones históricas y características sociales, políticas y económicas de los países receptores y oferentes de la inmigración, México y China en este caso. Cambios internos e internacionales han impactado en forma significativa los flujos migratorios de chinos a México.

2. Las relaciones establecidas con los gobiernos a nivel estatal y nacional en México. Instituciones empresariales, culturales y de aprendizaje del chinomandarín, por ejemplo, han recibido una excelente acogida, logrando una importante presencia y difusión de sus actividades y vínculos con instituciones y contrapartes en forma significativa. Uno de los casos más representativos es el Instituto "Confucio» y sus cinco sedes ${ }^{23}$ en México, convertido en uno de los países de América Latina con mayor número de sedes.

3. Pareciera ser - aunque debiera profundizarse en el futuro- que la procedencia y los niveles educativos y de ingresos han cambiado significativamente a lo largo de la historia de la migración china a México: desde los trabajadores agrícolas y mineros en el norte de México en la primera parte del siglo XX, a una clase empresarial y de comerciantes y un creciente flujo de empleados

${ }^{23}$ Las distintas sedes son: Instituto "Confucio» de la Universidad Nacional Autónoma de México; Instituto "Confucio» de la Universidad Autónoma de Nuevo León; Instituto «Confucio» de la Universidad Autónoma de Chihuahua; Instituto «Confucio» de la Universidad Autónoma de Yucatán e Instituto «Confucio» de la Ciudad de México-Huaxia. 
con mayor grado educativo hacia corporaciones chinas ubicadas en las afueras de la Ciudad de México.

El sector académico, conjuntamente con funcionarios, empresarios y contrapartes en China, debería en el futuro mejorar la calidad de la información existente, para contribuir a sentar las bases para una relación bilateral México-China mucho más profunda, habida cuenta del papel que las instituciones chinas en México podrían jugar para la consolidación en todos los ámbitos de la relación bilateral. Con base en el aporte de este documento, el análisis de la «nueva inmigración» china a México en el último lustro aparece particularmente interesante; instituciones como el Centro de Estudios de Enseñanza para Extranjeros (CEPE) de la UNAM, con más de 250 estudiantes chinos por bimestre, por ejemplo, reflejan esta nueva heterogeneidad de la inmigración china. El profundo e intenso intercambio entre las instituciones públicas chinas - en México y en China-y un grupo de instituciones empresariales y académicas chinas también es de interés, con el objetivo de conocer formas de funcionamiento, jerarquías, resultados esperados, etcétera, aunque seguramente con altas barreras y fuertes restricciones a la información. Por último, sería de gran relevancia enriquecer la diversa conformación territorial de comunidades chinas en México y destacar sus diferencias, tal y como se hizo inicialmente para los casos del Distrito Federal, Mexicali y Chiapas. El preliminar universo de instituciones de inmigrantes chinos contribuye a definir futuros territorios de investigación.

\section{Bibliografía}

Alcántara, C., M. Solís, O. Ojeda et al. (2009), "Los chinos que llegaron para quedarse», El Financiero (Sección Informe Especial), viernes 20 de marzo, p. 18.

Cauich Carrillo, Fredy (1998), «La comunidad china en el estado de Sonora. La migración china a Sonora y a los Estados Unidos de América (1882-1934)», tesis de licenciatura, México, UAM-I.

$140 \frac{\text { PRIMER SEMESTRE } 2016}{\text { MIGRACIÓN Y DESARROLLO NÚM. } 26}$ 
Centro de Estudios China-México (Cechimex) (2009), Directorio de instituciones y empresas chinas en la Ciudad de México 2009, México, Cechimex.

(2013), Directorio de empresas chinas y de instituciones mexicanas vinculadas con China en la Ciudad de México 2013, México, Cechimex.

Cinco Basurto, Mónica (1999), "Más allá de las fronteras: Los chinos en la Ciudad de México", tesis de maestría, México, UAM-I.

Dussel Peters, Enrique (2014), «La inversión extranjera directa de China en América Latina: 10 casos de estudio", México, Red ALC-China, UDUAL y UNAM/Cechimex.

Gómez Izauierdo, Jorge (1992), "El movimiento antichino en México (1871-1934)», en Problemas del racismo y del nacionalismo durante la Revolución Mexicana, México, INAH.

GonZÁlez FÉLIX, Maricela (1990), «El proceso de aculturación de la población de origen chino en la ciudad de Mexicali», Cuadernos de Ciencias Sociales, núm. 7.

Instituto Nacional de Estadística, Geografía e Informática (INEGI) (2010), Censo General de Población y Vivienda, México, INEGI.

Instituto Nacional de Migración (INM) (2009), Síntesis 2009. Estadística Migratoria, México, Secretaría de Gobernación/InM.

(2010), Síntesis 2010. Estadística Migratoria, México, Secretaría de Gobernación/INM.

(2011), Sintesis 2011. Estadística Migratoria, México, Secretaría de Gobernación/INM.

(2012), Sintesis 2012. Estadística Migratoria, México, Secretaría de Gobernación/INM.

(2013), Sintesis 2013. Estadística Migratoria, México, Secretaría de Gobernación/INM.

Jiménez Macías, Carlos (2012), "40 años de relaciones diplomáticas entre México y China», en Enrique Dussel Peters, 40 años de la relación entre México y China. Acuerdos, desencuentros y futuro, México, unAm/Cechimex, CICIR y Cámara de Senadores.

JACoues, Leo M. (1981), "Chinese Merchants in Sonora, 1900-1931», en Luz Ma. Martínez Montiel (ed.), Asiatic Migrations in Latin America, México, Colmex.

LAU, Rebeca (2010), "Mami: My Grandmother's Journey», Chinese Canadian Historical Society of British Columbia, vol. y núm.

LI, Jimmy (2012). "La Cámara (General) de Empresarios Chinos en México», En Enrique Dussel Peters (coord.), 40 años de la relación entre México y China. Acuerdos, desencuentros y futuro, México, unAm/Cechimex, CiCir y Cámara de Senadores. 
MarTínez Marín, Ricardo (1995), "La migración china en el estado de Tamaulipas, (1900-1940)», tesis de maestría en Historia, México, UAM-Iztapalapa.

Martínez Rivera, Sergio E. (2012), "Los empresarios chinos en México y la Ciudad de México: contexto histórico y actualidad», Revista Matices, Facultad de Estudios Superiores Aragón de la UnAm, 7 (18), mayo-agosto.

Monitor de la Manufactura Mexicana (2015), Monitor de la Manufactura Mexicana, 11. México, Cechimex/unam, pp. 50-54.

Portes, Alejandro (2015), «Immigration, Transnationalism, and Development: The State of the Question", en Alejandro Portes y Patricia Fernandez-Kelly, The State and the Grassroots: Immigrant Transnational Organizations in Four Continents. Nueva York, Berghahn Books.

Portes, Alejandro y Zhou Min (2012), «Transnationalism and Development: Mexican and Chinese Inmigrant Organizations in the United States», Population and Development Review, 38 (2).

Portes, Alejandro, Cristina Escobar y Alexandria Walton Radfordd (2007), «Inmigrant Transnational Organizations and Development: A Comparative Study», International Migration Review, 41 (primavera).

Puig Llano, Juan (1992), Entre el río Perla y el Nazas. La china decimonónica y sus braceros emigrantes. La Colonia china de Torreón y la matanza de 1911, México, Conaculta.

Qıv, Xiaoqi (2014), "China, profundización integral de la reforma y sus relaciones con México", Cuadernos de Trabajo del Cechimex, 3.

RéÑIQUE, Gerardo (2003), «Región, raza y nación en el antichinismo sonorense. Cultura regional y mestizaje en el México posrevolucionario», en Aarón Grageda Bustamante, Seis expulsiones y un adiós. Despojos y exclusiones en Sonora. México, Universidad de Sonora y Plaza y Valdés.

Velásouez Morales, Catalina (2005), "Diferencias políticas entre los inmigrantes chinos del noroeste de México (1920-1930). El caso de Francisco L. Yuen", Historia Mexicana, 55 (2).

Xu, Shicheng (2007), "Los chinos a lo largo de la historia de México», en Enrique Dussel Peters y Yolanda Trápaga Delfín (coord.), China y México: implicaciones de una nueva relación, Mexico, La Jornada Ediciones, Fundación Friedrich Ebert, Itesm y UNAM/Cechimex.

YangYang, Zhou (2014), "Análisis de la situación de los inmigrantes chinos en México y perspectivas», tesis de grado, China, University of International Business and Economics.

$142 \frac{\text { PRIMER SEMESTRE } 2016}{\text { MIGRACIÓN Y DESARROLLO NÚM. } 26}$ 
Zhou, Min y Lee Rennie (2015), «Traversing Ancestral and New Homelands: Chinese Immigrant Transnational Organizations in the United States», en A. Portes y P. Fernández-Kelly, The State and the Grassroots: Immigrant and Trasnational Organizations in Four Continents, Nueva York, Berghan Books. 
